\title{
DESIGN AND IMPLEMENTATION OF 10 BIT, 2MS/s SPLIT SAR ADC USING 0.18um CMOS TECHNOLOGY
}

\author{
Kalmeshwar N. Hosur ${ }^{1}$, Dr. Girish V. Attimarad ${ }^{2}$, Dr. Harish M. Kittur ${ }^{3}$ \\ ${ }^{1}$ Department of Electronics \& Communication Engg., S.D.M. College of Engineering \& \\ Technology, Dharwad, Karnataka, India \\ ${ }^{2}$ Department of Electronics \& Communication Engg., Dayanand Sagar College of \\ Engineering, Kumarswamy Layout, Bangalore, Karnataka, India \\ ${ }^{3}$ School of Electrical Sciences, VIT University,Vellore, Tamilnadu, India
}

\begin{abstract}
This paper focuses on Design and Implementation of 10 Bit, 2MS/s successive approximation Register (SAR) Analog to digital converter (ADC) using Split DAC architecture. This SAR ADC architecture is designed and simulated using GPDK 0.18um CMOS technology. It consists of different blocks like sample and hold, comparator, Successive Approximation Register (SAR) and Split Digital to analog converter $(D A C)$. For each block of SAR ADC power is calculated. DAC is an important component within the SAR $A D C$. The charge redistribution DAC in a Split capacitor configuration has a total capacitance which is 96.87\% smaller compared to a conventional binary weighted design. Hence Split DAC gives an optimized architecture and it consumes less power. Optimized design of DAC architecture ensures the accuracy of the components, which improves the performance of SAR ADC. Comparator constructed from resistances, capacitance and dependent voltage sources instead of MOS transistors. Dynamic range for SAR ADC using split DAC is $60.19 \mathrm{~dB}$. The supply voltage is $1.2 \mathrm{~V}$. The total Power consumed by SAR ADC using Split array DAC is $95.65114 u \mathrm{~W}$ and SAR ADC using binary weighted capacitor DAC is $211.19084 u \mathrm{~W}$.
\end{abstract}

\section{KEYWORDS}

Successive Approximation, Analog-to-Digital converter, Digital-to-Analog converter, Split array, Charge redistribution

\section{INTRODUCTION}

Successive approximation register (SAR) analog to digital converters (ADCs) have attracted more attention because of low power, excellent power efficiency, scalability and characteristics of digital nature. In the literature survey there are various kinds SAR topologies such as binaryweighted SAR, C-2C SAR, split SAR, etc. In general, their principle of working is based on the use of a binary search algorithm to estimate the digital equivalent of an input analog signal. SAR ADCs using binary weighted DAC present a good linearity performance but suffer from exponential reliance of the total capacitance on resolution which results in low sampling speed, and large area occupation. On the converse, much higher speed is provided by C-2C SAR ADCs 
International Journal of VLSI design \& Communication Systems (VLSICS) Vol.6, No.3, June 2015

but introduce non-linearity issue because of the parasitic capacitances at the intermediate nodes which limit their resolution below 8 bits. The resolution drawback of C-2C SAR ADCs is eliminated by split SAR ADCs while maintaining their speed advantage by reducing the number of intermediate nodes. Fundamentally, the split SAR ADC consists of two capacitor arrays most significant bit (MSB) array and least significant bit (LSB) array connected by a bridge capacitor. The total weight of the LSB array must be equal to the weight of the lowest-bit capacitor of the MSB array in order for the two capacitor arrays have the same scaling. This requires the attenuation capacitor to have a fractional value, which can be difficult to match when the design is implemented. Therefore, the linearity of the ADC is considerable reduced. In [1], to increase the linearity, an attenuation capacitor with integer capacitance value is used. An attenuation capacitor is added to the LSB array to keep the correct scaling. In addition, in order to reduce the input loading capacitance, one unit capacitor is added to the MSB array and the input is only sampled onto the bottom plates of the MSB array. To calibrate the weight mismatch between the lowest-bit capacitor of the MSB array and the capacitors of the LSB array a Capacitor DAC mismatch calibrator is designed [1] [6].

One serious issue for SAR ADCs is the capacitor mismatches that result from process variations and device parasitic. It normally limits the ADC linearity to less than 10-bit. The capacitor mismatch is minimized by increasing the size of the capacitor; however, this slows down the conversion speed and unfortunately increases the chip area and power. A much better way to alleviate the capacitor mismatch issue is to perform capacitor mismatch calibration [2] [3]. On one hand, in very high sampling rate (200-500 MS/s) applications, medium-resolution SAR ADCs are increasingly used. On the other hand, in low energy radios and biomedical applications, ultra low power and low-frequency SAR ADCs are being used. In several cases, a significant part toward the total power consumption of the SAR ADC is due to the capacitive digital-to-analog converter. This has brought to force the challenge of further reducing the power consumption of the DAC. Many studies have been performed on reducing the switching power of the DAC [4]. In SAR ADCs the binary-weighted capacitive DAC is more widely used. But, the exponential increase in the capacitance of the DAC array with the resolution, which imposes larger consumption of switching energy, area and settling time. The split capacitive DAC is a valuable alternatives, which has been recently reconsidered for medium resolution. Its key drawback lies in the parasitic capacitors that destroy the desired binary ratio of the capacitive DAC array, thus degrading the conversion linearity. However, the split structure can become suitable for a medium-resolution applications by using the metal-insulator-metal capacitor or/and DAC mismatch calibrations. On the other hand, the switching sequences of the DAC array are directly correlated with the conversion linearity, where the conventional charge-redistribution switching results in worse conversion linearity and extra energy losses [5].

In a SAR ADC, the capacitive DAC, comparator and SAR logic are the main sources of energy consumption. The digital energy consumption reduced by the technology innovation while the power consumption of comparator and capacitive DAC is restricted by the noise and matching requirements. The switching energy is significantly determined by the capacitor switching scheme mainly in the capacitive DAC [7]. The split SAR ADC offers the excellent trade-off in terms of area, speed, and power consumption [8]. The capacitive digital-to-analog converter dominates the overall power consumption compared with a digital control circuit and comparator in SAR analog-to-digital converter [9]. 
International Journal of VLSI design \& Communication Systems (VLSICS) Vol.6, No.3, June 2015

This paper describes design and implementation of 10-bit, 2MS/s split SAR ADC using 0.18um CMOS technology. Section 2 discusses the SAR ADC review. Section 3 discusses the basics of SAR ADCs and Modifications. Section 4 explains the implementation of split DAC and split SAR ADC using Cadence. Section 5 contains experimental results and Section 6 contains conclusions.

\section{SAR ADC REVIEW}

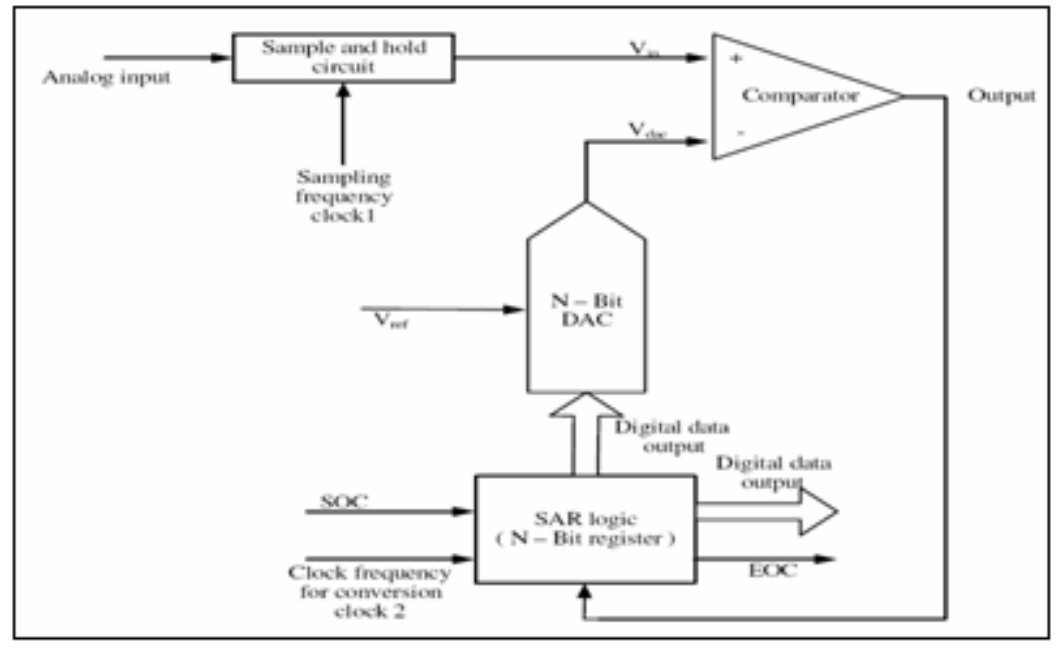

Figure 1. N-Bits SAR ADC Architecture

The block diagram of SAR-ADC is as shown in Fig. 1. It consists of sample and hold circuit, successive approximation register, N-bit capacitive DAC and high speed comparator. The fundamental principle of data conversion is based on successive approximation algorithm and is as given in (1) [10], the MSB bit sets $\mathrm{V}_{\text {ref }}$ to 0.5 . The next bit attenuates $\mathrm{V}_{\text {ref }}$ to 0.25 and so on, finally giving zero, once the analog signal value becomes equal to the sum of the attenuated $V_{\text {ref }}$ values. This approximation is the heart of any given SAR converter.

$\mathrm{V}_{\text {analog }}-\left\{\mathrm{V}_{\text {ref }}\left[\mathrm{b}_{0} 2^{-1}+\mathrm{b}_{1} 2^{-2}+---\mathrm{b}_{\mathrm{N}-1} 2^{-\mathrm{N}}\right]\right\}=0$.

\section{BASICS OF SAR ADC AND MODIFICATIONS}

\subsection{Sample and Hold circuit}

The sample and hold circuit design is a key aspect in any high resolution, high speed ADC process, as the conversion process depends only on the instantaneous voltage developed across the hold capacitor. The simplest form of a sample and hold circuit design for a sinusoidal input, and the circuit behaviour can be graphically shown in Fig. 2 and Fig. 3 respectively. It consists of a MOSFET switch with a finite $\mathrm{ON}$ resistance $\mathrm{R}$, a hold capacitor of value $\mathrm{C}$ and a clock signal controlling the ON/OFF time of MOS switch. 
International Journal of VLSI design \& Communication Systems (VLSICS) Vol.6, No.3, June 2015

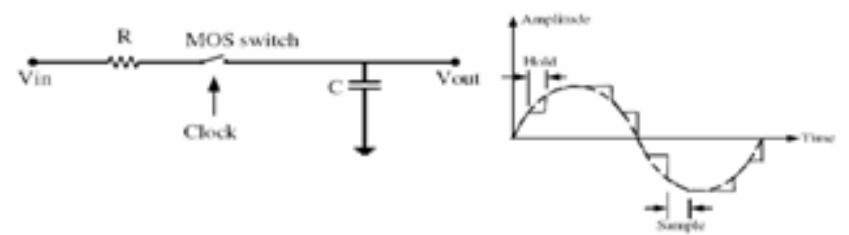

Figure 2. Sample and Hold Circuit Figure 3. Response to sinusoidal I/P

\subsection{Charge Redistribution DAC}

Fig. 4 shows a charge redistribution DAC is a parallel array of binary-weighted capacitors, and $2^{\mathrm{N}} \mathrm{C}$ is the total capacitance. All capacitors are discharged firstly. The digital signal switches each capacitor to either ground or $V_{\text {ref }}$, causing the output voltage, $V_{\text {out }}$, to be a function of the voltage division between the capacitors [11].

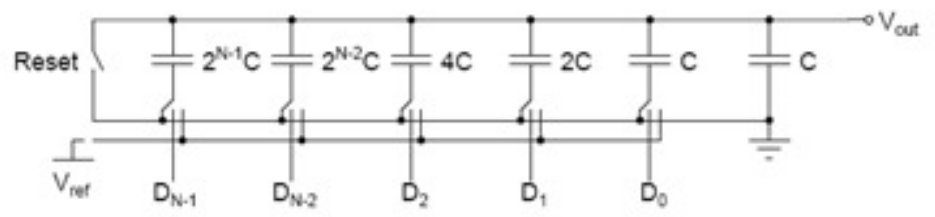

Figure 4. A Charge-redistribution DAC

The capacitor array totals $2^{\mathrm{N}} \mathrm{C}$. Thus, if the MSB is high and the remaining bits are low, then a voltage divider occurs between the MSB capacitor and the rest of the array. The analog output voltage, $V_{\text {out }}$, becomes:

$$
\begin{aligned}
V_{\text {out }} & =V_{\text {ref }} \cdot \frac{2^{N-1} C}{\left(2^{N-1}+2^{N-2}+2^{N-3}+\ldots+4+2+1+1\right) C} \\
& =V_{r e f} \cdot \frac{2^{N-1} C}{2^{N} C}=\frac{V_{r e f}}{2}
\end{aligned}
$$

Which confirms the fact that the MSB changes the output of a DAC by $1 / 2 V_{\text {ref. }}$. Fig. 5 shows the equivalent circuit under this condition.

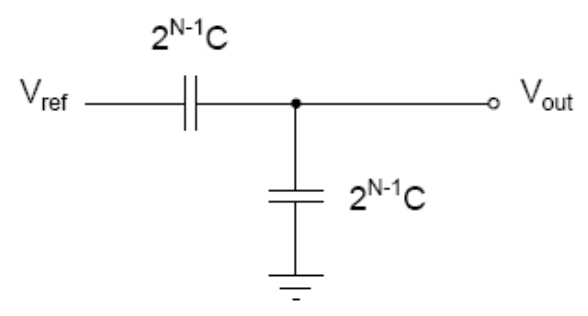

Figure 5. Equivalent circuit with the MSB $=1$, and all other bits set to zero

The ratio between $V_{\text {out }}$ and $V_{\text {ref }} d u e$ to each capacitor can be generalized to:

$V_{\text {out }}=\frac{2^{k} C}{2^{N} C} \cdot V_{r e f}=2^{k-N} \cdot V_{r e f}$ 
International Journal of VLSI design \& Communication Systems (VLSICS) Vol.6, No.3, June 2015

Where it is assumed that the $k$-th bit, $D_{k}$, is one and all other bits are zero. Superposition can then be used to find the value of $V_{\text {out }}$ for any input word by:

$$
V_{\text {out }}=\sum_{k=0}^{N-1} D^{k} 2^{k-N} . V_{r e f}
$$

\subsection{Split array DAC}

The charge-redistribution architecture is very much accepted because of its simplicity and relative good accuracy. While a linear capacitor is required, high resolution in the 10- to 12-bit range can be achieved. However, as the resolution increases, the size of the MSB capacitor becomes a main concern. For example if the unit capacitor, $C$, were $0.5 \mathrm{pF}$, and a 12 -bit DAC were to be designed, the MSB capacitor would need to be:

$\mathrm{C}_{\mathrm{MSB}}=2^{\mathrm{N}-1} \cdot 0.5 \mathrm{pF}=1024 \mathrm{pF}$

A split array technique used to reduce the size of capacitors. A 6-bit example of the array is shown in Fig. 6. This architecture is slightly different from the charge-redistribution DAC shown in Fig. 4 in that the output is taken off a different node and an additional attenuation capacitor is used to separate the array into a LSB array and a MSB array. Note that the MSB $\left(\mathrm{D}_{5}\right)$ corresponds to the rightmost switch and the LSB $\left(\mathrm{D}_{0}\right)$ now corresponds to the leftmost switch [11].

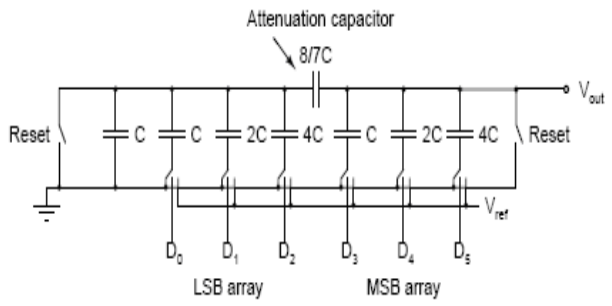

Figure 6. A Charge-redistribution DAC using a split array

The value of the attenuation capacitor can be found by:

$\mathrm{C}_{\mathrm{att}}=($ Sum of the LSB array capacitors / sum of the MSB array capacitors $) * \mathrm{C}$

Where the sum of the MSB array equals the sum of LSB capacitor array minus $C$. The value of the attenuation capacitor should be such that the series combination of the attenuation capacitor and the LSB array, assuming all bits are zero, equals $C$. To prove this a derivation is made; refer to formula (7) [12]. The output voltage is defined as the attenuation factor times the LSB bits plus the MSB bits times the reference voltage. The attenuation factor is a capacitive divider between the attenuation capacitor and the sum of the LSB capacitors. One can see that with some manipulation this is equal to formula (4).

$$
\begin{aligned}
V_{\text {out }}= & \left(\frac{\frac{2^{N / 2}}{2^{N / 2}-1}}{\frac{2^{N / 2}}{2^{N / 2}-1}+2^{N / 2}} \sum_{k=0}^{N / 2-1} D_{k} 2^{k-N / 2}+\sum_{k=N / 2}^{N-1} D_{k} 2^{k-N}\right) \cdot V_{r e f} \\
& =\left(\frac{1}{2^{N / 2}} \sum_{k=0}^{N / 2-1} D_{k} 2^{k-N / 2}+\sum_{k=N / 2}^{N-1} D_{k} 2^{k-1}\right) \cdot V_{r e f}
\end{aligned}
$$




$$
\begin{aligned}
& =\left(\frac{2^{N / 2}}{2^{N / 2}} \cdot \sum_{k=0}^{N / 2-1} D_{k} 2^{k-N}+\sum_{k=N / 2}^{N-1} D_{k} 2^{k-N}\right) \cdot V_{r e f} \\
& =\left(\sum_{k=0}^{N / 2-1} D_{k} 2^{k-N}+\sum_{k=N / 2}^{N-1} D_{k} 2^{k-N}\right) \cdot V_{r e f} \\
& =\sum_{k=0}^{N-1} D_{k} 2^{k-N} \cdot V_{r e f}
\end{aligned}
$$

\subsection{Comparator}

The introduction of the Application Specific Integrated Circuits has created the need to simulate complex circuits which include both analog and digital functions on the same chip. The circuit simulation becomes quite difficult because of couple of problems. Firstly, mixed mode simulators are required for the combined analog and digital functions on the same chip. Secondly, the large number of active devices per chip, significantly increases the required simulation time and increases the problem of convergence. Hence, macro-modeling addresses the second concern by reducing the complexity of the circuit. Macro-models use simplified simulation elements mathematical functions to define the behavior of a simulation model. Macro-modeling techniques are the only practical method of modeling some complex circuitry. Macro-functions are used in practice to reduce modeling-time and make simulations run faster and converge better [13]. The macro model of comparator constructed from resistances, capacitance and dependent voltage source is shown in Fig. 7 instead of transistors. It reduces the simulation time and power consumption.

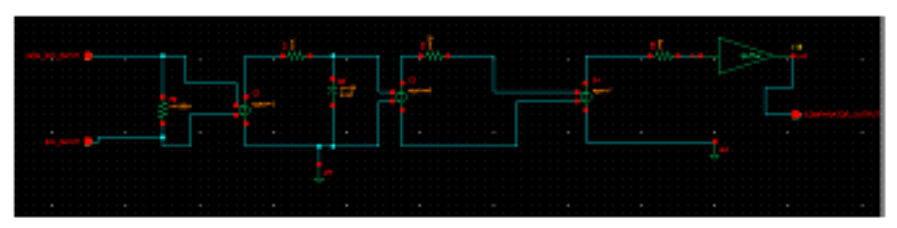

Figure 7. Comparator.

\subsection{SAR LOGIC DESIGN}

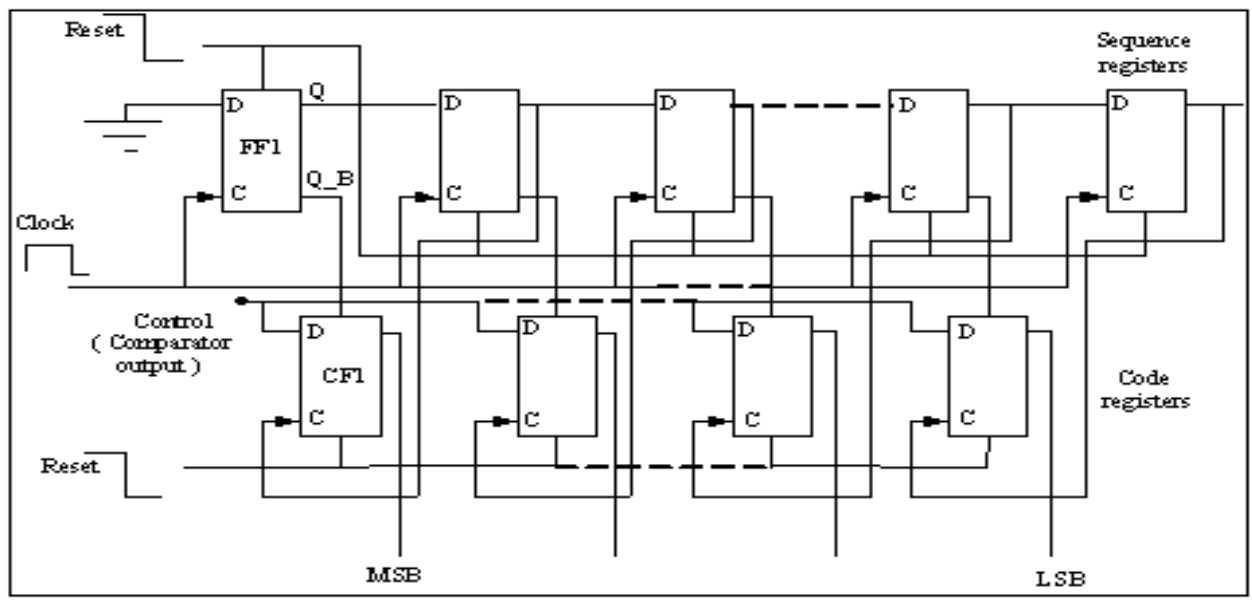

Figure 8. SAR Logic [14] 
International Journal of VLSI design \& Communication Systems (VLSICS) Vol.6, No.3, June 2015

The schematic of the SAR logic is as shown in Fig. 8. Firstly the reset line goes low, and controls set line of flip-flop1 [FF1] and reset lines of all other sequencer flip-flops. The same reset signal also controls the reset line of code register flip-flops. Output Q and output Q_B of FF1 are set to 1 and 0 respectively. Q_B also controls set line of code register flip-flop1 [CF1]. Hence the CF1 output is forced to 1 . This is the MSB bit and the weight for voltage full scale range VFSR/2. It should be noted that, since sequence register is reset initially, the set input of all the code registers flip- flops except CF1 is at logic 1 . Hence all the other code register output states are logic 0 . We get a sequence $\mathrm{MSB}=1$ and all others set to 0 . The analog equivalent of this weight will be generated by the DAC. When reset goes high and clock is triggered, (notice that D input of FF1 sequencer array is grounded-logic 0) Q becomes 0 and FF2 outs logic High. This low to high transition of FF2 triggers or clocks the code register flip- flop CF1 to store control bus value (comparator output) to its output. When clock runs further, the code register flip-flop retains the set value as FF2 output goes to zero (D-flip flop positive edge triggered). This process is repeated for each of the flip-flops until after N-clock cycles a high state comes out of sequencer flip-flop controlling the code register least significant bit [LSB] flip-flop.

\section{SYSTEM IMPLEMENTATION}

\subsection{Implementation of Split DAC}

The schematic of 10 bit split DAC architecture is shown in Fig.9. In this a unit capacitance of $2.5 \mathrm{fF}$ is used. Attenuation capacitor of $2.5806 \mathrm{fF}$ is used to separate LSB capacitor array and MSB capacitor array. On MSB side input bits are $\mathrm{D}_{9}, \mathrm{D}_{8}, \mathrm{D}_{7}, \mathrm{D}_{6}, \mathrm{D}_{5}$ and LSB side input bits are $\mathrm{D}_{4}, \mathrm{D}_{3}$, $\mathrm{D}_{2}, \mathrm{D}_{1}$, and D0. On MSB side each capacitor value is divided into two half capacitors connected in parallel and one half of the capacitance is connected to ground potential and other half capacitance is connected to corresponding input bit as shown in Fig. 10. This is done in order to reduce the voltage on the top node of the LSB side of the SPLIT DAC. This increasing of node voltage happens when DAC is connected in integrated SAR ADC. The symbol view of split DAC is shown in Fig. 11.

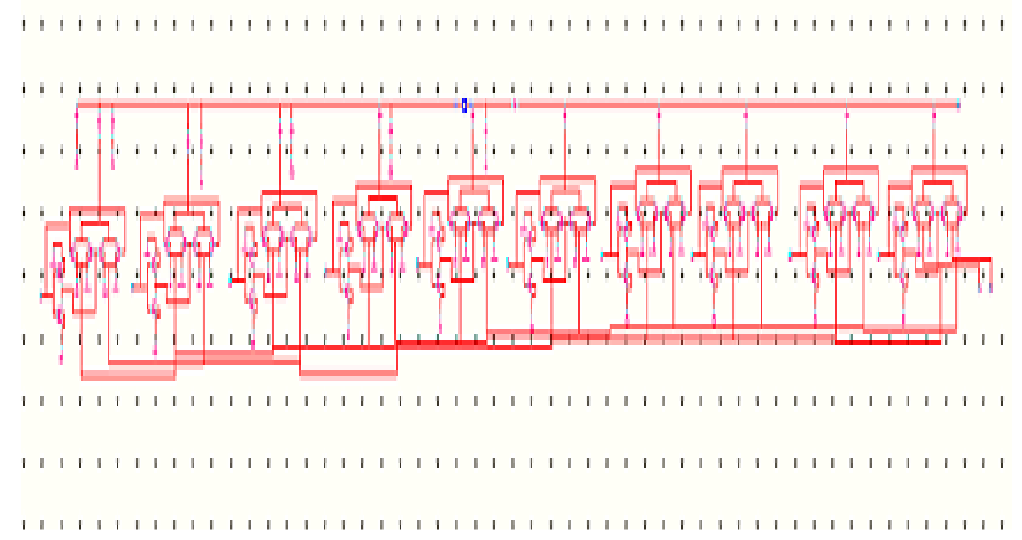

Figure 9. Schematic of 10 Bit Split DAC 
International Journal of VLSI design \& Communication Systems (VLSICS) Vol.6, No.3, June 2015

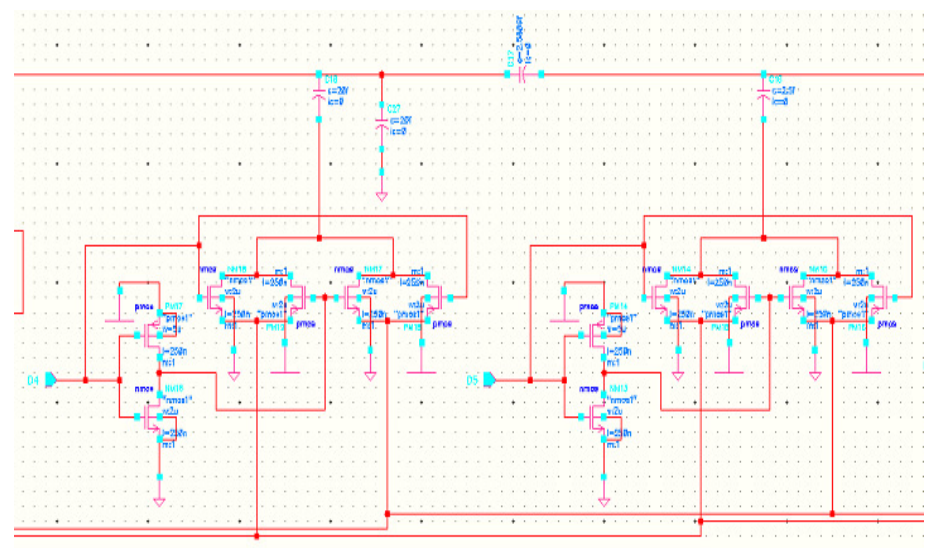

Figure 10. Circuit of Split DAC for 1Bit on LSB and 1 Bit on MSB side.

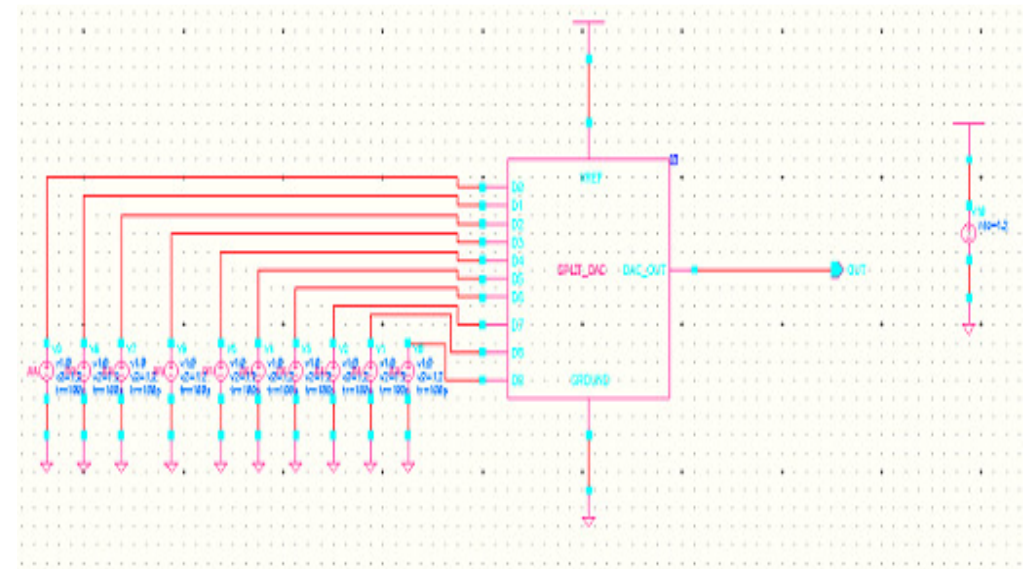

Figure 11. Symbol of Split DAC

\subsection{Implementation of SAR ADC}

The 10-bit SAR ADC using split DAC architecture is as shown in Fig. 12. The Fig. 12 consists of sample and hold circuit, comparator, SAR block and split DAC array. For this circuit analog signal of peak to peak amplitude of $1.2 \mathrm{~V}$ with an offset of $600 \mathrm{mV}$ and frequency of $100 \mathrm{KHz}$ is applied. The sampling signal of clock period is $1 \mathrm{us}$ and high voltage of $1.2 \mathrm{~V}$ and low voltage of $0 \mathrm{~V}$ is given. The clock signal of SAR block is $50 \mathrm{~ns}$, high voltage level of $1.2 \mathrm{~V}$ and low voltage level of $0 \mathrm{~V}$. 
International Journal of VLSI design \& Communication Systems (VLSICS) Vol.6, No.3, June 2015

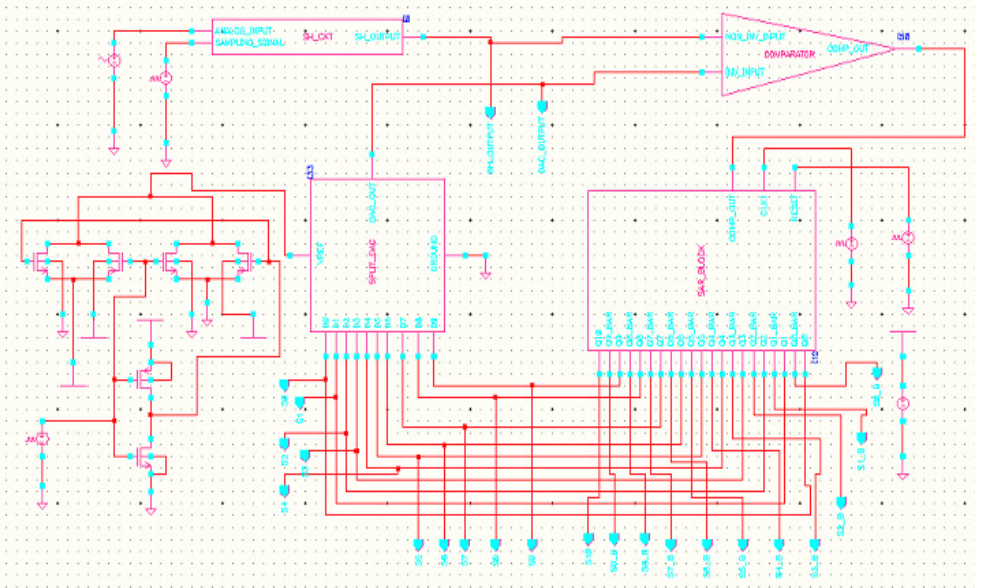

Figure 12. SAR ADC schematic using Split DAC

\section{EXPERIMENTAL RESULTS}

The simulation results of 10 bit split DAC architecture is shown in Fig. 13. Table I mentions 10 input samples and corresponding outputs in hex form of SAR ADC using split DAC. The simulation results of 10-Bit SAR ADC using split DAC are shown in Fig. 14. Table II \& Table III mention power consumed in watts and in $\%$ by each block of SAR ADC using split array DAC and binary weighted DAC respectively. Total power consumed by SAR ADC using split DAC is $95.65114 \mathrm{uW}$ and SAR ADC using binary weighted DAC is $211.1908 \mathrm{uW}$.

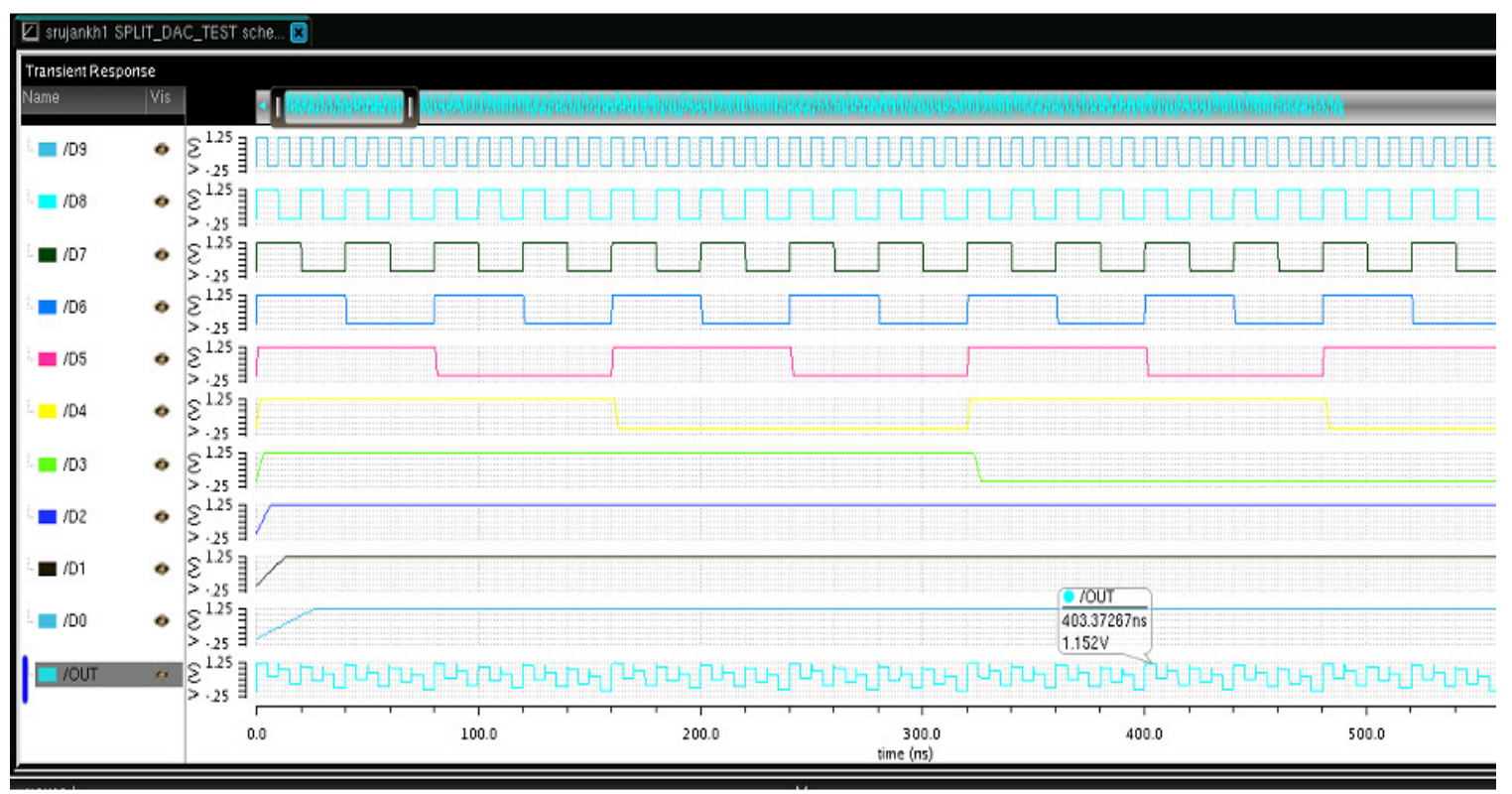

Figure 13. output waveforms of Split DAC 
International Journal of VLSI design \& Communication Systems (VLSICS) Vol.6, No.3, June 2015

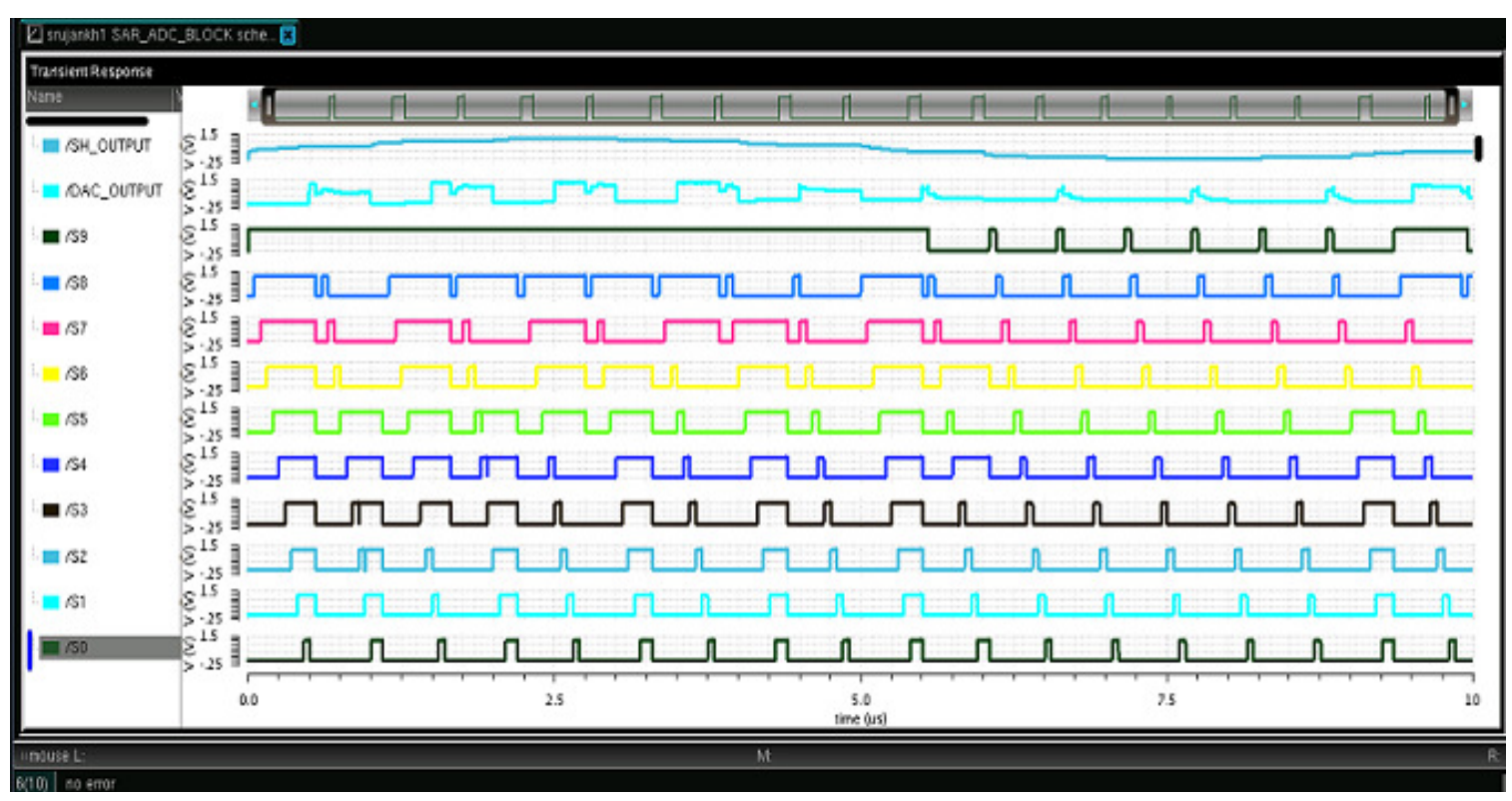

Figure 14 Output waveforms of Split DAC ADC

TABLE I: Input sine wave Samples and corresponding output of SAR ADC using split DAC.

\begin{tabular}{|c|c|c|c|}
\hline Time & $\begin{array}{c}\text { Sample and } \\
\text { Hold circuit } \\
\text { output }\end{array}$ & $\begin{array}{c}\text { Split DAC } \\
\text { output }\end{array}$ & $\begin{array}{c}\text { SAR ADC } \\
\text { OUTPUT in } \\
\text { HEX }\end{array}$ \\
\hline 1us & $754.4 \mathrm{mV}$ & $706.1 \mathrm{mV}$ & $23 \mathrm{E}$ \\
\hline $2 \mathrm{us}$ & $1.095 \mathrm{~V}$ & $1.06 \mathrm{~V}$ & 338 \\
\hline 3us & $1.19 \mathrm{~V}$ & $1.174 \mathrm{~V}$ & 370 \\
\hline 4us & $1.089 \mathrm{~V}$ & $920.1 \mathrm{mV}$ & $2 \mathrm{C} 0$ \\
\hline 5us & $802.1 \mathrm{mV}$ & $801.4 \mathrm{mV}$ & 200 \\
\hline 6us & $418.4 \mathrm{mV}$ & $362.0 \mathrm{mV}$ & 051 \\
\hline 7us & $115.3 \mathrm{mV}$ & $245.7 \mathrm{mV}$ & 004 \\
\hline 8us & $18.9 \mathrm{mV}$ & $210.1 \mathrm{mV}$ & 018 \\
\hline 9us & $113.9 \mathrm{mV}$ & $188.9 \mathrm{mV}$ & 040 \\
\hline 10us & $411.6 \mathrm{mV}$ & $460.03 \mathrm{mV}$ & 100 \\
\hline
\end{tabular}


International Journal of VLSI design \& Communication Systems (VLSICS) Vol.6, No.3, June 2015

TABLE II: Power consumption of each block of SAR ADC using Split DAC.

\begin{tabular}{|l|l|l|}
\hline $\begin{array}{c}\text { Different blocks } \\
\text { of SAR ADC }\end{array}$ & Power consumed & \multicolumn{1}{|c|}{$\begin{array}{c}\text { \% of Power } \\
\text { consumped }\end{array}$} \\
\hline $\begin{array}{l}\text { Sample and Hold } \\
\text { Circuit }\end{array}$ & $21.4487 \mathrm{nW}$ & 0.0224 \\
\hline Comparator & $10.4799 \mathrm{uW}$ & 10.9563 \\
\hline $\begin{array}{l}\text { Successive } \\
\text { Approximation } \\
\text { Logic }\end{array}$ & $63.4359 \mathrm{uW}$ & 66.3200 \\
\hline $\begin{array}{l}\text { Split Digital to } \\
\text { Analog } \\
\text { Converter }\end{array}$ & $17.4600 \mathrm{uW}$ & 18.2538 \\
\hline $\begin{array}{l}\text { Power Saving } \\
\text { Circuit }\end{array}$ & $4.2539 \mathrm{uW}$ & 4.4473 \\
\hline $\begin{array}{l}\text { Complete SAR } \\
\text { ADC }\end{array}$ & $95.65114 \mathrm{uW}$ & 100 \\
\hline
\end{tabular}

TABLE III: Power consumption of each block of SAR ADC using binary weighted DAC.

\begin{tabular}{|l|l|l|}
\hline $\begin{array}{l}\text { Different blocks of } \\
\text { SAR ADC }\end{array}$ & Power consumed & \multicolumn{1}{|c|}{$\begin{array}{c}\text { \% of Power } \\
\text { consumped }\end{array}$} \\
\hline $\begin{array}{l}\text { Sample and Hold } \\
\text { Circuit }\end{array}$ & $21.4487 \mathrm{nW}$ & 0.0101 \\
\hline Comparator & $10.4799 \mathrm{uW}$ & 4.9622 \\
\hline $\begin{array}{l}\text { Successive } \\
\text { Approximation } \\
\text { Logic }\end{array}$ & $63.4359 \mathrm{uW}$ & 30.0372 \\
\hline $\begin{array}{l}\text { Binary Weighted } \\
\text { capacitor Digital } \\
\text { to Analog } \\
\text { Converter }\end{array}$ & $132.9997 \mathrm{uW}$ & 62.9760 \\
\hline $\begin{array}{l}\text { Power Saving } \\
\text { Circuit }\end{array}$ & $4.2539 \mathrm{uW}$ & 2.0142 \\
\hline $\begin{array}{l}\text { Complete SAR } \\
\text { ADC }\end{array}$ & $211.1908 \mathrm{uW}$ & 100 \\
\hline
\end{tabular}


International Journal of VLSI design \& Communication Systems (VLSICS) Vol.6, No.3, June 2015

\section{CONCLUSION}

The designed 10 Bit, 2MS/s Successive Approximation ADC using Split DAC architecture is simulated using GPDK 0.18um CMOS technology and compared power consumption of this architecture with SAR ADC using binary weighted DAC architecture. Dynamic range for this architecture is $60.19 \mathrm{~dB}$. The charge redistribution DAC in a Split capacitor configuration has a total capacitance which is $96.87 \%$ smaller compared to a conventional design. Hence split array DAC occupies smaller area. The power consumed by SAR ADC using binary weighted DAC is 211.1908uW and Split DAC is $95.65114 \mathrm{uW}$. Hence power consumed by SAR ADC using split capacitor configuration is less. Power consumed by each block of SAR ADC using split DAC and SAR ADC using binary weighted DAC are mentioned in Table II and Table III. Power consumption of binary weighted DAC is $132.9997 \mathrm{uW}$ and split DAC is $17.46 \mathrm{uW}$.

\section{ACKNOWLEDGEMENT}

We thank the management, Principal, Staff of S.D.M College of Engineering and Technology, Dharwad, Karnataka, India, for encouraging us for this research work.

\section{REFERENCES}

[1] Anh Trong Huynh1, Hoa Thai Duong2, Hoang Viet Le1, and Efstratios Skafidas1,2,1National ICT Australia, Australia 2Department of Electrical and Electronics Engineering, University of Melbourne, VIC, 3010, Australia, "Design and Implementation of an 11-bit 50-MS/s Split SAR ADC in $65 \mathrm{~nm}$ CMOS", 978-1-4799-3432-4/14/\$31.00@2014IEEE.

[2] Anh Trong Huynh1, Hoa Thai Duong2, Hoang Viet Le1, and Efstratios Skafidas1,2, 1National ICT Australia, Australia 2Department of Electrical and Electronic Engineering, University of Melbourne, VIC, 3010, Australia, "Design and Implementation of an 11-bit 50-MS/s Split SAR ADC in $65 \mathrm{~nm}$ CMOS", 978-1-4799-3432-4/14/\$31.00 @2014 IEEE.

[3] Hyunsoo Ha, Seon-Kyoo Lee, Byungsub Kim, Member, IEEE, Hong-June Park, Senior Member, IEEE, and Jae-Yoon Sim, Senior Member, IEEE, "A 0.5-V, 1.47 $\mu \mathrm{W}$ 40-kS/s 13-bit SAR ADC With capacitor Error Compensation", IEEE TRANSACTIONS ON CIRCUITS AND SYSTEMS-II: EXPRESS BRIEFS, VOL. 61, NO. 11, NOVEMBER 2014.

[4] Arindam Sanyal, Student Member, IEEE, and Nan Sun, Member, IEEE, “An Energy-Efficient Low Frequency-Dependence Switching Technique for SAR ADCs", IEEE TRANSACTIONS ON CIRCUITS AND SYSTEMS-II: EXPRESS BRIEFS,VOL.61, NO. 5, MAY 2014.

[5] Yan Zhu, Chi-Hang Chan, U-Fat Chio, Sai-Weng Sin, Seng-Pan U, Rui Paulo Martins, and Franco Maloberti, "Split SAR ADCs: Improved linearity with power and speed optimization", IEEE TRANSACTIONS on VERY LARGE SCALE INTEGRATION (VLSI) SYSTEMS, VOL. 22, NO.2, FEBRUARY 2014.

[6] Ji-Yong UM, Yoon -Jee Kim, Eun-Woo Song, Jae-Yoon Sim, Member, IEEE, and Hong-June Park, Member IEEE, “ A Digital Domain Calibration of Split-Capacitor DAC for a Differential SAR ADC Without Additional Analog Circuits", IEEE TRANSACTIONS On Circuits and Systems-I: REGULAR PAPERS, VOL.60, NO.11,NOVEMBER 2013

[7] Yue $\mathrm{Wu} * \mathrm{Xu}$ Cheng * and Xiaovang Zeng, State Key Laboratory of ASIC \& System Fudan University Shanghai 200433, China, "A Split- Capacitor Vcm-based Capacitor-switching Scheme for Low power SAR ADCs", 978-1-4673-5762-3/13/\$31.00@2013 IEEE.

[8] Anh Trong Huynh*, Hoa Thai Duong\# , Hoang Viet Le*, and Efstratios\#**National ICT Australia, \#Department of Electrical and Electronics Engineering, University of Melbourne, VIC 3010, Australia, "Design of a Capacitive DAC Mismatch Calibrator for Split SAR ADC in 65nm CMOS", Asia-Pacific Microwave Conference Proceedings, 2013. 
International Journal of VLSI design \& Communication Systems (VLSICS) Vol.6, No.3, June 2015

[9] Liangbo Xie, Guangjun Wen, Jiaxin Liu and Yao Wang, "Energy-efficient hybrid capacitor switching scheme for SAR ADC”, ELECTRONICS LETTERS 2nd January 2014, Vol. 50, No. 1 pp. 22-23.

[10] Phillip E. Allen and Douglas R. Holberg, "CMOS Analog Circuit Design", Second Edition, New York Oxford, Oxford University Press 2004.

[11] Jacob Baker, Harry W.Li and David E. Boyce, "CMOS Circuit Design, Layout, and Simulation", IEEE Press, Prentice Hall of India Private Limited, New Delhi 2000.

[12] Frank B. Boschker, "Design of a 12 bit $500 \mathrm{MS} / \mathrm{s}$ standalone charge redistribution Digital-to-Analog Converter", University of Twente, Msc. Thesis January 2008.

[13] Ray Kendall, "Modular macro modeling techniques for spice simulators", PE, SDC Inc, Intuitive Research and Technology Corp (Huntsville, A1), EDN 93, March 72002.

[14] Krishnachandran K Nair, "Low power Analogue to Digital converter for Power Management IC's", M.Sc project on System Level Integration, 2005.

\section{AUTHORS}

Kalmeshwar N. Hosur, working as Assistant Professor in the department of Electronics and Communication Engineering, SDMCET, Dharwad, Karnataka, India. Received B.E (Electronics and Communication Engineering) from Karnataka University, Dharwad and M.Tech (VLSI Design and Embedded Systems) from VTU, Belgaum. Currently pursuing Ph.D in the field of VLSI. Areas of interests are Digital and Analog VLSI Design, Embedded Systems, data converter architectures.

Dr. Girish V. Attimarad, working as Professor and HOD, department of Electronics and Communication Engineering, Dayanand Sagar College of Engineering and Technology, Bangluru, Karnataka, India. Recived B.E (Electronics and Communication Engineering) from Gulbarga University, Gulbarga, India, M.E (Digital Electronics) from Karnataka University, Dharwad, India and Ph.d (Mirowave Communication ) from Delhi Unversity, Delhi, India.

Dr. Harish M. Kittur, working as Professor and HOD School of VLSI Design, VIT, Vellore, Tamilnadu, India. Received M.Tech (Solid State Technologies) from IIT Madras, India and Ph.D from RWTH Aachen, Germany. Areas of Interests are VLSI Design, Solid State Technology, and Nanotechnology.
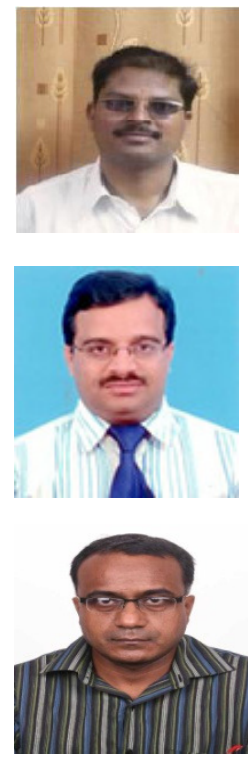